\title{
Incorporação de pó de agregados reciclados de concreto como fíler em misturas asfálticas
}

\author{
Incorporating powder from recycled concrete \\ aggregates as filler in hot mixture asphalt
}

\author{
Juan Gabriel Bastidas-Martínez ${ }^{1}$, Juan Carlos Ruge Cardenas ${ }^{1}$, \\ Liosber Medina García $^{2}$, Robinson Andrés Giraldo Zuluaga ${ }^{2}$
}

\footnotetext{
${ }^{1}$ Programa de Engenharia Civil, Universidade Católica de Colômbia, CP: 110111, Bogotá D.C., Colômbia.

${ }^{2}$ Curso de Engenharia de Transportes, Faculdade de Ciências e Tecnologia, Universidade Federal de Goiás, UFG, C EP: 74968-755, Aparecida de Goiânia, GO, Brasil.

e-mail: jgbastidas@ucatolica.edu.co; jcruge@ucatolica.edu.co; lmedina@ufg.br; zuluaga@ufg.br
}

\section{RESUMO}

A geração e o gerenciamento de resíduos sólidos industriais consideram-se uma problemática ambiental no mundo. Vários estudos têm se concentrado na utilização de resíduos da construção civil no âmbito da pavimentação. O objetivo deste artigo é avaliar o uso do pó de agregados reciclados de concreto RFPA (Recycled Fine Aggregates Powder) no Concreto Betuminoso Usinado a Quente (CBUQ). Para tal fim, se fabricaram três tipos de misturas asfálticas: a mistura de referência com materiais convencionais e dois misturas de estudo com substituição do $3 \%$ e $6 \%$ do filer natural pelo RFAP (composição total da massa da mistura). Avaliou-se a resistência sob carga monotônica das misturas (Ensaio Marshall e Tração Indireta-RT) e a resistência ao desgaste Cântabro. Uma análise de variância (ANOVA) foi realizada para verificar se os resultados obtidos eram estatisticamente significativos. Ao substituir a 3\% do fíler da granulometria pelo pó RFAP, obtevese um comportamento mecânico satisfatório sob a ação das cargas monotônicas, embora houvesse aumento da perda de massa no ensaio Cântabro. Os resultados evidenciam que a utilização do RFAP da forma proposta é viável desde o ponto de vista técnico e ambiental, por permitir uma forma de disposição final segura de resíduos.

Palavras-chave: Fíler, RFPA, Ensaio Marshall, Resistência à tração indireta.

\begin{abstract}
The generation and management of industrial solid waste is a global environmental problem. Several studies have focused on the use of civil construction waste in the paving field. The main goal of this paper is to evaluate the use of recycled fine aggregate powder (RFAP) in a Hot Mix Asphalt (HMA). For this purpose three types of asphalt mixtures were produced: the reference mixture with conventional materials and two study mixes with replacement of $3 \%$ and $6 \%$ of natural filer by RFAP (total composition of the mixture mass). The monotonic load strength of the mixtures (Marshall Test and Indirect Traction-RT) and the resistance to Cantabro wear was evaluated. When replacing $3 \%$ of the total composition of the mixture mass with RFAP powder a satisfactory mechanical behavior was obtained under monotonic loads, although there was an increase in mass loss in the Cantabro test. The results show that the use of RFAP in the proposed way is feasible from a technical and environmental point of view, as it allows a safe final disposal of waste.
\end{abstract}

Keywords: Filler, RFPA, Marshall test, tensile strength.

\section{INTRODUÇÃO}

O crescimento da população mundial tem elevado a demanda de materiais adequados para a construção e manutenção de infraestruturas civis. Os recursos naturais, cada vez mais limitados, são a principal preocupação para atingir os patamares do desenvolvimento sustentável. Rochas naturais são matérias primas não renováveis e usadas em larga escala em misturas asfálticas. Desta forma, nos últimos tempos, tem-se proposto alternativas para ajudar a solucionar esse problema. Uma dessas soluções é o uso de resíduos de construção e demolição (RCD). Esse material é composto principalmente de resíduos de concreto, morteiros, tijolos, ma- 
deira, cerâmica, fundição, vidro, plásticos e solos de escavação, entre outros [1, 2]. Entre os resíduos citados, aqueles provenientes do concreto são denominados de RCA pelas suas siglas em inglês (Recycled Concrete Aggregate). No mundo, grandes quantidades de RCD e RCA são produzidas. Na Europa, reportou-se a geração de 320 milhões de toneladas em 2016 [3]. Na china, a geração atingiu 2.36 milhões de toneladas de RCA entre os anos 2003 a 2013. [4]. Na Colômbia, as principais cidades geram mais de 100.000 toneladas de RCD por dia [5]. Desta forma, esses materiais são um dos principais resíduos gerados no mundo que afeta negativamente a preservação ambiental.

Uma opção viável para diminuir os volumes destes resíduos é a reutilização para a construção e restauração de novas obras de pavimentação, já que esse tipo de empreendimento demanda uma grande quantidade de materiais para sua execução. Uma das dificuldades no aproveitamento do RCD é a heterogeneidade de suas características, o que pode dificultar a determinação das propriedades mecânicas. Para isso, vários estudos têm se concentrado no entendimento do uso de agregados de RCD para a construção de camadas em estruturas de pavimentação [6-11]. Um problema ambiental que pode surgir com o uso desses resíduos é a dissolução de pó no ar e na água, causando aumento do pH nos sistemas de águas subterrâneas e afetando a vegetação. No caso especifico do uso de misturas asfálticas do tipo CBUQ (Concreto Betuminoso Usinado a Quente), a incorporação de pó não gera problemas devido às características impermeáveis da material resultante [12].

Vários pesquisadores têm estudado o uso de agregados reciclados do concreto RCA na elaboração de misturas asfálticas [13-18]. Esses estudos se concentraram na substituição em massa das frações grossas, finas ou totais dos agregados naturais pelos RCAs. Isso é feito com a finalidade de fornecer uma alternativa de disposição final dos resíduos de concreto ambientalmente segura. Os resultados apresentados na literatura são viáveis do ponto de vista técnico, pois atendem aos parâmetros volumétricos e de resistência contemplados nas metodologias de projeto. Porém, no desempenho do comportamento mecânico existem discrepâncias. Por um lado, alguns pesquisadores apresentam maior rigidez, maior resistência à deformação permanente e maior resistência à danos por umidade induzida das misturas com RCA quando comparadas com as misturas convencionais [1, 17, 19-22] Por outro, alguns autores relatam resultados contrários [15, 23-26]. Isso é amplamente atribuído à origem, características físicas, composição química e mineralógica dos RCAs.

Existem poucos trabalhos reportados na literatura que realizem substituição do fíler convencional por pó do RCA [1, 27]. As evidências nesses casos demonstraram uma maior rigidez da mistura causada pelo menor volume de vazios e pelas interações físico-químicas superficiais entre o cimento residual da massa RCA e o asfalto. Isso resultou em uma maior resistência à deformação permanente e resistência à fadiga, no momento que foram comparadas com as misturas de controle com fíller convencional. Além disso, os autores concluem que a maior quantidade de partículas de fíler com o uso de RCA, pode gerar uma maior trabalhabilidade do material, no que se refere à fabricação e compactação da mistura. Apesar dos resultados contraditórios, os autores concluíram que a utilização do RCA é viável, devido ao atingimento dos parâmetros de resistência mínimos utilizados para a dosagem de concretos asfálticos para capas de rolamento ou de camadas binder [28].

Na literatura, pode-se observar a aplicação de frações miúdas dos agregados RCA em misturas asfálticas [12, 29], nas quais avaliaram o comportamento mecânico de misturas com substituição do fíler da granulometria por pó de agregados finos reciclados (chamado de RFAP pelas suas siglas em inglês - Recycled Fine Aggregates Powder). Os resultados encontrados indicam que a presença de RFAP no concreto asfáltico melhora a resistência à umidade, à deformação permanente e à fadiga. No entanto, os autores reportaram que o uso do RFAP na mistura pode causar a diminuição no desempenho a baixas temperaturas devido à elevada rigidez.

Neste contexto, o presente trabalho tem como objetivo avaliar o uso do pó de RFAP como fíler no CBUQ por meio dos ensaios de carga monotônica (Estabilidade Marshall e resistência à tração) e de resistência ao desgaste Cântabro numa primeira etapa. Posteriores etapas procuraram avaliar o comportamento mecânico da mistura asfáltica ante a ação de cargas dinâmicas e estáticas. A hipótese do estudo se fundamentou na grande quantidade de cimento Portland presente no RFAP, que pode contribuir para o desenvolvimento de propriedades pozolânicas na mistura, aumentando possivelmente a coesão e adessividade entre os agregados e o ligante asfáltico.

\section{MATERIAIS E MÉTODOS}

A metodologia geral seguida no trabalho é apresentada na Figura 1, na qual são mostradas as principais informações sobre as avaliações experimentais realizadas para o entendimento do efeito da adição de fíler de pó de RFAP na mistura asfáltica. Em um primeiro momento, foram realizados ensaios de caracterização dos materiais separadamente, sendo verificado o cumprimento das especificações segundo as respectivas normas. Posteriormente, passou-se para o processo de dosagem da mistura de referência, ponto de partida para a con- 
fecção das misturas com RFAP. Assim, avançou-se com a fase de caracterização mecânica para verificar o desempenho das misturas. Por último, foram feitas análises estatísticas dos resultados para interpretar as diferenças entre elas.

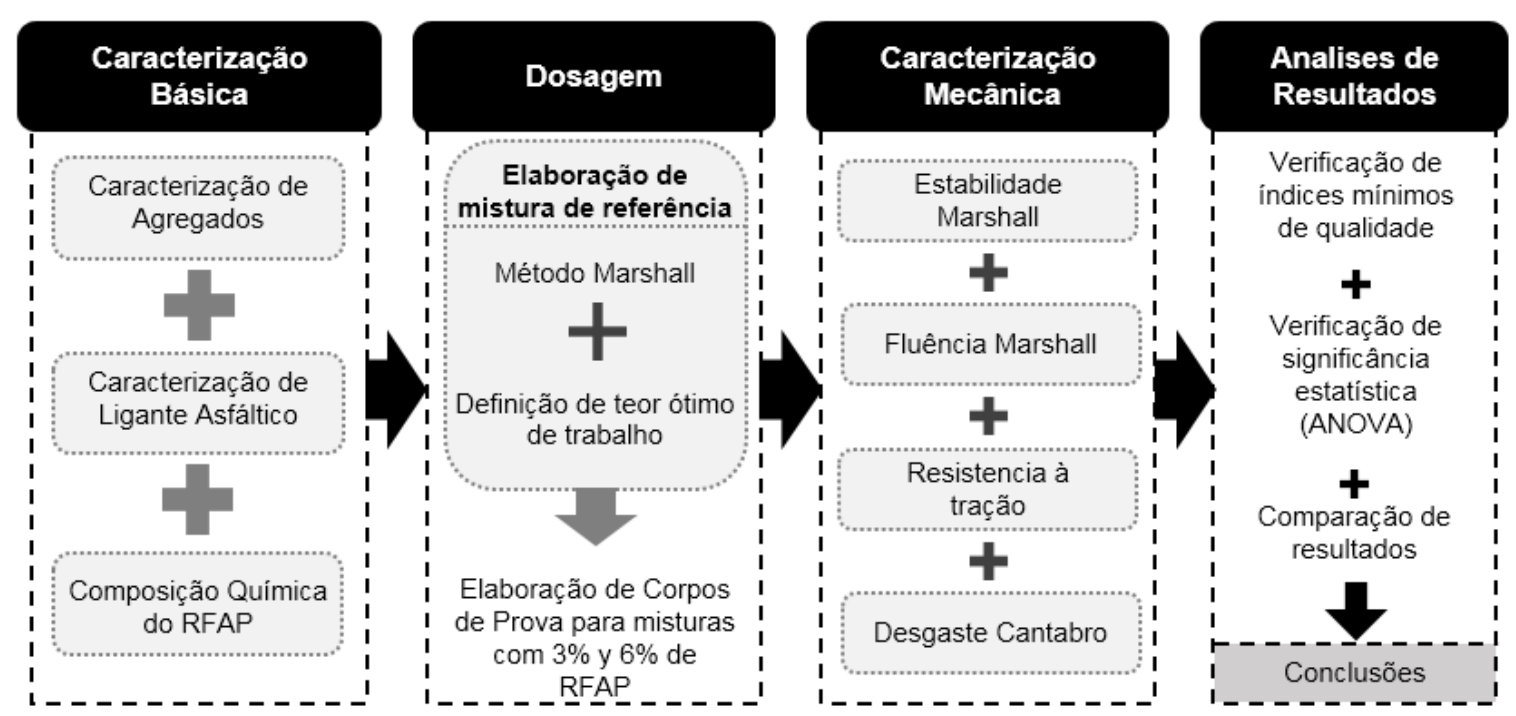

Figura 1: Esquema da metodologia de trabalho.

\subsection{Materiais}

O RCA foi obtido numa das plantas de coleta autorizada pelo Instituto de Desenvolvimento Urbano (IDU) da cidade de Bogotá, Colômbia. O RCA foi triturado com almofariz até atingir a condição de pó (RFAP), ou seja, o equivalente a 100\% do material passa pela peneira №200 $(0.075 \mathrm{~mm})$. O resultado da massa específica real foi de $3.11 \mathrm{~g} / \mathrm{cm}^{3}$ conforme a norma ASTM C188 [30]. O resultado pode ser atribuído a grande quantidade de cimento proveniente do RCA. Realizou-se a caracterização química, mediante o ensaio de espectroscopia de fluorescência de raios-X para obter a composição química elementar, conforme é apresentada na Tabela 1. Segundo os resultados, é possível observar a presença de óxido de silício $\left(65.7 \%\right.$ de $\left.\mathrm{SiO}_{2}\right)$, óxido de cálcio (23.3\% de $\mathrm{CaO}$ ), óxido de alumínio (4.6\% $\mathrm{Al}_{2} \mathrm{O}_{3}$ ) e outros componentes em menor proporção. Esses resultados são coerentes com os obtidos por Pasandín e Pérez [26]. Esse aspecto pode ser benéfico no aumento da adesividade do concreto asfáltico.

Tabla 1: Composição química do pó de RFAP.

\begin{tabular}{l|c|c}
\hline MINERAL & COMPOSIÇÃO QUímICA & \% EM MASSA \\
\hline Óxido de sílice & $\mathrm{SiO}_{2}$ & 65,70 \\
\hline Óxido de alumínio & $\mathrm{Al}_{2} \mathrm{O}_{3}$ & 4,60 \\
\hline Óxido de cálcio & $\mathrm{CaO}$ & 23,30 \\
\hline Óxido de ferro & $\mathrm{Fe}_{2} \mathrm{O}_{3}$ & 3,80 \\
\hline Magnesita & $\mathrm{MgCO}_{3}$ & 2,10 \\
\hline Óxido de sulfura & $\mathrm{SO}_{3}$ & 0,50 \\
\hline
\end{tabular}

O agregado convencional (AC) utilizado na produção das misturas asfálticas também procedeu da cidade de Bogotá, Colômbia. A Tabela 2 apresenta a caracterização física segundo as especificações do INVIAS [31]. De acordo com os resultados, é possível evidenciar que os critérios para a fabricação de CBUQ são atendidos, assim, utilizou-se os valores médios da granulometria da faixa MDC-19 exigidos pelo Artigo 450 da especificação supracitada (ver Figura 2). 
Tabla 2: Propriedades Físicas dos agregados AC

\begin{tabular}{l|c|c|c|c}
\hline ENSAIO & NORMA & UNIDADE & RESULTADO & ESPECIFICAÇÃO \\
\hline Abrasão "Los Ángeles" & AASHTO T 96-02 [32] & $\%$ & 19,5 & Máx. 25-35 \\
\hline Micro-Deval & AASHTO T 327 [33] & $\%$ & 22,3 & Máx. 20-25 \\
\hline $\begin{array}{l}\text { Índice de } \\
\text { achatamento/alongamento }\end{array}$ & NLT-354/91 [34] & $\% / \%$ & $19 / 14$ & \\
\hline Faces fraturadas & ASTM D5821 [35] & $\%$ & 85,2 & Min. 85 \\
\hline $\begin{array}{l}\text { Durabilidade dos Agregados } \\
\text { pelo Uso do Sulfato de Sódio } \\
\text { ou Magnésio }\end{array}$ & AASHTO T 104-99 [36] & $\%$ & 13,34 & Máx. 18 \\
\hline $\begin{array}{l}\text { Agregado graúdo - adesividade } \\
\text { a ligante betuminoso }\end{array}$ & DNER-ME 078-94 [37] & ---- & Cumpre & ---- \\
\hline Massa específica finos/absorção & AASHTO T 84-00 [38] & $----/ \%$ & $2,60 / 3,1$ & \\
\hline Massa específica graúdos/absorção & AASHTO T 85-91 [39] & $----/ \%$ & $2,64 / 1,8$ & -- \\
\hline
\end{tabular}

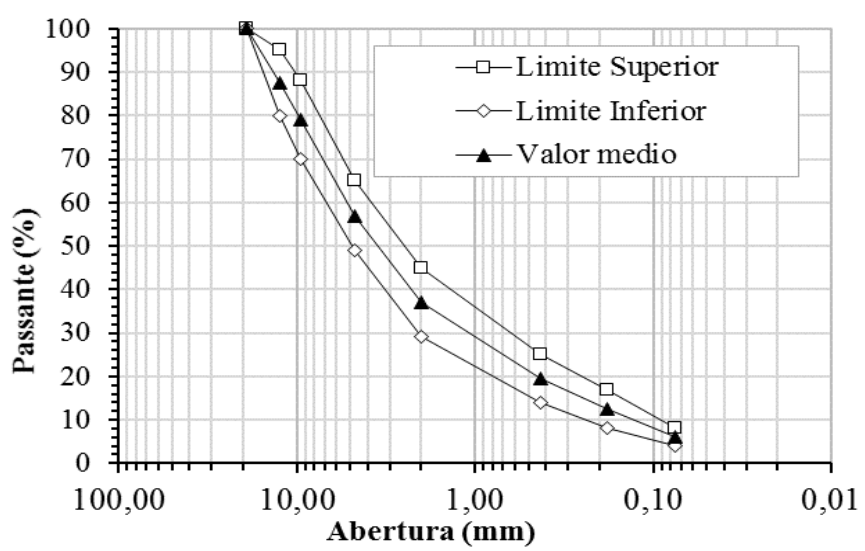

Figura 2: Especificação granulométrica da mistura CBUQ (MDC-19) INVIAS 2013.

Tabla 3: Propriedades Físicas do CAP 60-70

\begin{tabular}{|c|c|c|c|c|c|}
\hline \multirow{2}{*}{ ENSAIO } & \multirow{2}{*}{ NORMA } & \multirow{2}{*}{$\begin{array}{l}\text { UNI- } \\
\text { DADE }\end{array}$} & \multicolumn{2}{|c|}{ ESPECIFICAÇÃO } & \multirow{2}{*}{ VALOR } \\
\hline & & & MIN. & MÁX. & \\
\hline \multicolumn{6}{|c|}{ ENSAIO SOBRE A AMOSTRA SEM ENVELHECIMENTO } \\
\hline Penetração $\left(25^{\circ} \mathrm{C}, 100 \mathrm{~g}, 5 \mathrm{~s}\right)$ & ASTM D5 / D5M [40] & $0.1 \mathrm{~mm}$ & 60 & 70 & 61,10 \\
\hline Ponto de Amolecimento & ASTM D36 [41] & ${ }^{\circ} \mathrm{C}$ & 48 & 54 & 52,00 \\
\hline Índice de Penetração & NLT 181/88 [42] & - & $-1,50$ & $+0,70$ & $-0,67$ \\
\hline Massa específica & $\begin{array}{c}\text { AASHTO T228-04 } \\
{[43]}\end{array}$ & - & - & - & 1,022 \\
\hline Viscosidade dinâmica $\left(60^{\circ} \mathrm{C}\right)$ & AASHTO T-316 [44] & Poises & 1500 & - & 1750 \\
\hline Ponto de fulgor & ASTM D3143 [45] & ${ }^{\circ} \mathrm{C}$ & 230 & - & 338 \\
\hline Ductilidade $\left(25^{\circ} \mathrm{C}, 5 \mathrm{~cm} / \mathrm{min}\right)$ & ASTM D113 [46] & $\mathrm{cm}$ & 100 & - & $>105$ \\
\hline \multicolumn{6}{|c|}{ AMOSTRA APÓS RTFOT } \\
\hline Perda de massa & ASTM D2872 [47] & $\%$ & - & 0,8 & 0,1 \\
\hline Penetração (\% penetração amostra virgem) & ASTM D5 / D5M [40] & $\%$ & 55 & - & 81 \\
\hline Ponto de amolecimento & ASTM D36 - 95 [41] & ${ }^{\circ} \mathrm{C}$ & - & 8 & 7,5 \\
\hline
\end{tabular}

Os resultados da caraterização física do cimento asfáltico (CAP 60-70) são apresentados na Tabela 3, demonstrando que eles atendem os parâmetros de qualidade especificadas pelo INVIAS [31]. A caracteriza- 
ção do ligante asfáltico foi realizada em amostras sem envelhecimento e amostras envelhecidas no processo RTFOT (Rolling Thin-Film Oven Test), conforme a especificação da ASTM D2872 [47].

\subsection{Dosagem da mistura CBUQ de referência}

Foram elaboradas três misturas de CBUQ com Agregados Convencionais (AC) e cimento asfáltico de petróleo CAP 60-70. A primeira amostra foi composta com $100 \%$ do fíler natural e foi codificada como CBUQ de referência. A segunda e a terceira amostras foram fabricadas substituindo, de forma parcial e total, o correspondente a $50 \%$ e $100 \%$ do fíler natural pelo pó de RFAP, ou seja, 3\% e $6 \%$ da massa total respectivamente (CBUQ-3 RFAP, CBUQ-6 RFAP). A substituição parcial segue os lineamentos da especificação IDU-620-18 [48], que define uma substituição de 50\% do fíler tradicional. No caso da substituição total, o objetivo foi avaliar a possibilidade de um maior uso desse material alternativo em misturas asfálticas.

O projeto de dosagem das misturas foi realizado seguindo os preceitos da metodologia Marshall, segundo o Artigo 450 da especificação colombiana do Instituto Nacional de Vias [31]. Para a determinação do teor ótimo de asfalto da mistura CBUQ de referência, foram confeccionados 12 corpos de prova utilizando os AC e o CAP 60-70. Avaliou-se 4 teores prováveis de CAP correspondentes ao 4.5\%, 5.0\%, 5.5\% e $6.0 \%$ sobre o total da mistura. Para cada teor de asfalto, foram moldados três corpos de prova seguindo os padrões típicos deste tipo de procedimento [14, 49, 50]. Cada corpo de prova foi moldado com 1200 gramas de mistura CBUQ e dimensões aproximadas de $10 \mathrm{~cm}$ de diâmetro e $6.35 \mathrm{~cm}$ de altura. As temperaturas de fabricação da mistura e de compactação foram de $145^{\circ} \mathrm{C}$ e $155^{\circ} \mathrm{C}$, as quais foram obtidas pela curva da viscosidade do CAP 60-70. Os corpos de prova foram compactados com 75 golpes por ambas faces.

Finalmente, foram avaliados os parâmetros volumétricos, volume de vazios $(\mathrm{Vv})$, vazios no agregado mineral (VAM) e relação betume-vazios (RBV), e os parâmetros de resistência, estabilidade (E), fluência (F) e relação estabilidade/fluência (E/F). Desta forma foi definido o teor ótimo de asfalto para a mistura CBUQ de referência, o qual valor que foi mantido para a fabricação das demais misturas (CBUQ-3 RFAP e CBUQ-6 RFAP). Esta definição fundamenta-se no fato de que as partículas de RFAP possuem uma maior densidade que o material convencional, resultando numa mistura com um menor número de partículas de fíler, o que garante que o teor de asfalto da mistura de controle seja suficiente para revestir todas as partículas da mistura com RPAF. Além disso, a fixação do teor de asfalto não acrescenta custos econômicos para a fabricação de CBUQ, em termos de quantidade de ligante, focando o estudo na verificação do funcionamento dessa alternativa. Após a obtenção do teor ótimo de asfalto foram realizados outros corpos de prova para as fases seguintes. Além do descrito, foram determinadas a resistência à tração indireta (RT), a adesividade do ligante ao esqueleto mineral e a resistência ao desgaste Cântabro.

\subsection{Resistencia à tração indireta por compressão diametral estática - RT}

$\mathrm{O}$ ensaio RT consiste na aplicação de uma carga monotônica sobre um corpo de prova Marshall solicitado diametralmente no estado plano de deformação na temperatura de $25{ }^{\circ} \mathrm{C}$. A aplicação do carregamento foi realizada à velocidade de $50 \mathrm{~mm} / \mathrm{s}$. A determinação da $\mathrm{RT}$ em $\mathrm{kPa}$, é determinada por:

$$
R T=\frac{2000 P}{\pi t D}
$$

onde $\mathrm{P}$ corresponde à máxima carga de ruptura $(\mathrm{N}), \mathrm{h}$ e d a altura e o diâmetro em $(\mathrm{mm})$ respectivamente.

\subsection{Ensaio Cântabro}

O ensaio Cântabro foi desenvolvido para determinar o desgaste por abrasão de misturas abertas [51]. No entanto, no caso de misturas asfálticas densas, pode ser utilizado para avaliar a durabilidade e as propriedades de coesão. O percentual de abrasão é determinado num corpo de prova Marshall pela diferença de massa inicial e final, quando submetido a 300 revoluções do tambor da máquina de Los Angeles, a uma velocidade de 30 a $33 \mathrm{rpm}$ à temperatura de $25{ }^{\circ} \mathrm{C}$. No presente estudo, três amostras Marshall foram compactadas para cada mistura produzida e submetidas ao ensaio.

\subsection{Análise estatística}

Com o objetivo de avaliar se os resultados das misturas de RFAP têm diferenças significativas, em relação aos parâmetros volumétricos, mecânicos (Ensaio Marshall e RT) e de adesividade, com a mistura CBUQ de referência, foi aplicado um teste de análise de variância ANOVA adotando um nível de significância $(\alpha)$ de 0,05. Previamente, foram realizados testes de normalidade Shapiro-Wilk para verificar a distribuição dos parâmetros em discusão. 


\section{APRESENTAÇÃO E DISCUSSÃO DOS RESULTADOS}

A seguir são apresentados os resultados obtidos durante a execução da campanha experimental e suas respectivas análises. Inicialmente são apresentados os dados do processo de dosagem. Posteriormente, são feitas as comparações dos parâmetros mecânicos das misturas e, por último, os percentuais de abrasão.

\subsection{Dosagem da mistura de referência}

Os resultados da dosagem da mistura CBUQ de referência pela metodologia Marshall são apresentados na Tabela 4. O teor ótimo de asfalto é de 5,3\% segundo as especificações estabelecidas pelo INVIAS [31]. Para misturas CBUQ a especificação estabelece valores mínimos de $\mathrm{E}$ de $9 \mathrm{kN}$; F entre 2,0 e 4,0 mm; (E/F) entre 3,0 e 5,0 kN/mm; Vv entre 4,0\% e 6,0\%; VAM mínimo de $15,0 \%$ e RBV entre $65,0 \%$ e 75,0\%.

Tabela 4: Resultados da média de três corpos de prova para dosagem Marshall da mistura CBUQ.

\begin{tabular}{|c|c|c|c|c|c|c|c|c|}
\hline $\begin{array}{c}\text { ASFALTO } \\
(\%)\end{array}$ & $\begin{array}{c}\text { GMB } \\
\left(\mathrm{g} / \mathrm{cm}^{3}\right) \\
\end{array}$ & $\begin{array}{c}\text { DMT } \\
\left(\mathrm{g} / \mathrm{cm}^{3}\right)\end{array}$ & $\begin{array}{l}\text { VV } \\
(\%) \\
\end{array}$ & $\begin{array}{l}\text { VAM } \\
(\%)\end{array}$ & $\begin{array}{l}\text { RBV } \\
(\%)\end{array}$ & $\begin{array}{c}E \\
(k N)\end{array}$ & $\begin{array}{c}F \\
(\mathrm{~mm})\end{array}$ & $\begin{array}{c}E / F \\
(k N / m m)\end{array}$ \\
\hline 4,5 & 2,284 & 2,451 & 6,8 & 16,7 & 60,1 & 10,9 & 3,5 & 3,1 \\
\hline 5,0 & 2,309 & 2,433 & 5,1 & 16,2 & 69,3 & 12,3 & 3,7 & 3,3 \\
\hline 5,5 & 2,321 & 2,415 & 3,9 & 16,3 & 76,5 & 12,5 & 3,9 & 3,2 \\
\hline 6,0 & 2,338 & 2,398 & 2,5 & 16,4 & 83,4 & 11,1 & 4,0 & 2,8 \\
\hline
\end{tabular}

Com este teor ótimo de 5,3\% são moldados outros corpos de provas para estabelecer os valores de referência dos parâmetros volumétricos e mecânicos que serão comparados com as misturas elaboradas com RFAP. Conforme foi descrito na metodologia, as misturas de CBUQ com RFAP foram fabricadas com o mesmo teor de asfalto da mistura CBUQ de referência.

\subsection{Resultados de parâmetros volumétricos Dosagem da mistura de referência}

Comparando-se os parâmetros volumétricos (Vv e RBV) das misturas de CBUQ produzidas em função do teor de pó de RFPA, foi possível observar uma relação linear entre estes, conforme apresentado na Figura 3. Na medida em que o fíler foi substituído, houve aumento do volume de vazios com a consequente diminuição do RBV. Este comportamento pode ser atribuído ao menor número de partículas do fíler de pó de RFAP em referência ao fíler natural, o que pode ser justificado pelo valor elevado de massa específica real do pó de RFAP. Nesse sentido, a presença de teores de pó de RFAP aumenta o Vv e diminui o recobrimento das partículas (menor RBV) no interior da mistura de CBUQ. Outra razão do comportamento apresentado pode ser atribuída ao alto nível de absorção que apresentam os materiais provenientes do RCA, gerando menor recobrimento de asfalto sobre os agregados (diminuição de RBV) e, consequentemente, maior Vv, como reportado na literatura. De forma geral, pode-se evidenciar que a mistura CBUQ de referência e as misturas com substituição de fíler (CBUQ-3 RFAP e CBUQ-6 RFAP) atingiram os parâmetros para a fabricação de camadas de rolamento de pavimentos flexíveis segundo a especificação do INVIAS [31].

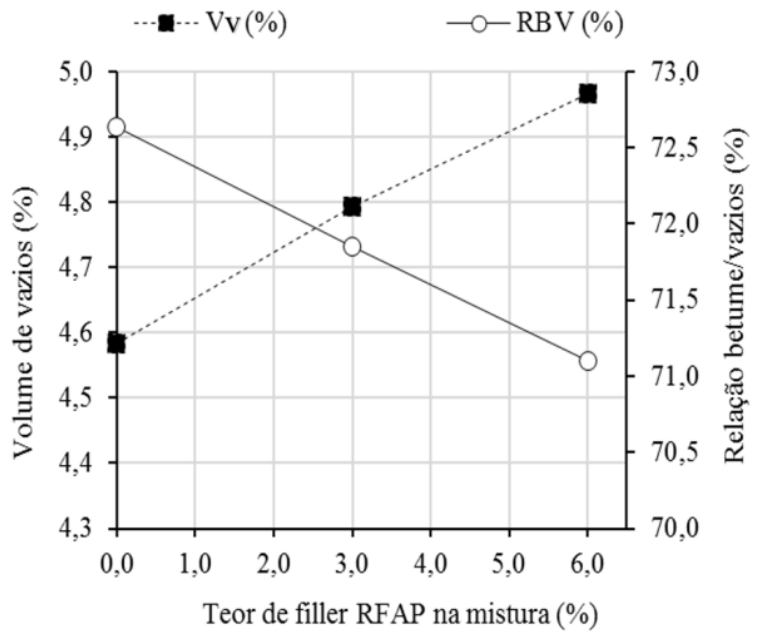

Figura 3: Parâmetros volumétricos das misturas. 
Os resultados dos parâmetros de resistência Marshall: (E, F e E/F) para as misturas são apresentados na Figura 4. Segundo os resultados, pode-se evidenciar que a mistura CBUQ-3 RFPA apresentou melhor comportamento devido ao aumento da $\mathrm{E}$ e E/F em relação à mistura CBUQ de referência. Esses aumentos foram gerados apesar do aumento de Vv e diminuição do RVB. Os resultados indicam um possível aumento um aumento da coesão interna das partículas, possivelmente pelas interações físico-químicas de superfície entre o fíler e o CAP 60-70. Nesse sentido, em coerência com os resultados de caracterização química do pó de RCA, a presença de óxido de silício $\left(65.7 \%\right.$ de $\left.\mathrm{SiO}_{2}\right)$ e óxido de cálcio $(23.3 \%$ de $\mathrm{CaO}$ ), pode ter contribuído ao aumento da rigidez [26]. Alguns trabalhos consultados na literatura especializada [52-54] concluíram que o uso de materiais ricos em conteúdo de sílica $\left(\mathrm{SiO}_{2}\right)$ aumenta a coesão entre o ligante asfáltico e os agregados. Isto pode impactar no aumento da rigidez, visto que o $\mathrm{SiO}_{2}$ é um material que apresenta alta dureza, resistência mecânica e pode permitir um melhor intertravamento no esqueleto mineral dos agregados. Os aumentos da rigidez foram maiores para a mistura CBUQ-3 RFAP que para a mistura CBUQ-6 RFAP, podendo estar relacionados à acomodação interna das partículas na mistura CBUQ. Para avaliar se os resultados dos parâmetros de resistência nas misturas com RFAP foram significativos com respeito à amostra CBUQ de referência, realizou-se análises de estatística descritiva (ANOVA) e a prova de normalidade de Shapiro-Wilk, conforme se apresenta na Tabela 5 .

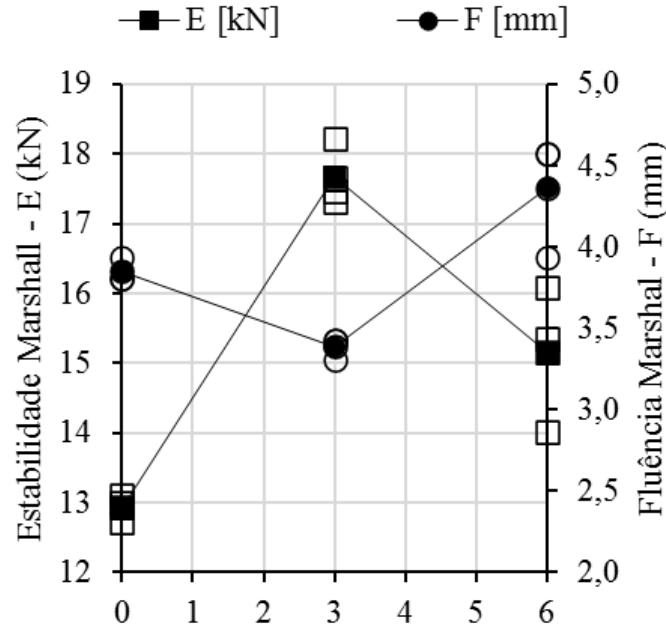

Teor de filler RAP na mistura (\%)

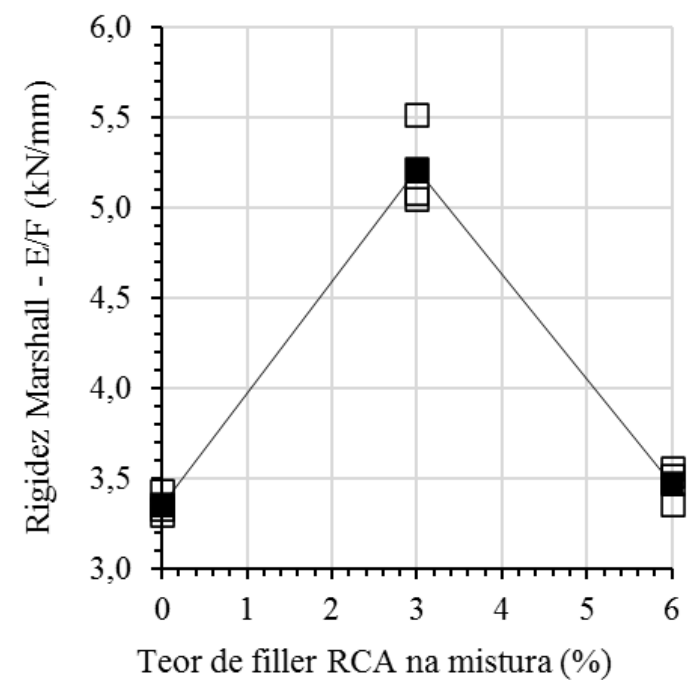

Teor de filler RCA na mistura (\%)

Figura 4: Variação da (a) E, F e; (b) E/F em função do teor de RAP.

Tabela 5: Estatística dos parâmetros mecânicos Marshall.

\begin{tabular}{c|c|c|c|c|c}
\hline PARÂMETRO & AMOSTRA & MÉDIA & DESVIO PADRÃO & P & NORMALIDADE SHAPIRO-WILK \\
\hline \multirow{4}{*}{$\mathrm{E}(\mathrm{kN})$} & CBUQ Referência & 12,91 & 0,20 & 0,86 & Sim \\
\cline { 2 - 6 } & CBUQ-3 RFPA & 17,66 & 0,48 & 0,28 & Sim \\
\cline { 2 - 6 } & CBUQ-6 RFPA & 15,15 & 1,04 & 0,69 & Sim \\
\hline \multirow{5}{*}{$\mathrm{F}(\mathrm{mm})$} & CBUQ Referência & 3,88 & 0,07 & 0,42 & Sim \\
\cline { 2 - 6 } & CBUQ-3 RFPA & 3,38 & 0,08 & 0,63 & Sim \\
\cline { 2 - 6 } & CBUQ-6 RFPA & 4,63 & 0,25 & 0,78 & Sim \\
\hline \multirow{3}{*}{ E/F $(\mathrm{kN} / \mathrm{mm})$} & CBUQ Referência & 3,36 & 0,07 & 0,68 & Sim \\
\cline { 2 - 6 } & CBUQ-3 RFPA & 5,22 & 0,26 & 0,14 & Sim \\
\cline { 2 - 6 } & CBUQ-6 RFPA & 3,48 & 0,11 & 0,36 & Sim \\
\hline
\end{tabular}

p: significância estatística

A partir dos resultados da Tabela 5, pode-se perceber que as misturas CBUQ-3 RFAP e CBUQ-6 RFAP conferiram um aumento na média da E de 36,7\% e 17,4\%, respectivamente, se comparadas com a mistura de referência. Estes incrementos foram estatisticamente significativos de acordo com os resultados da análise de variância ANOVA $\left(\mathrm{F}=36,82>\mathrm{F}_{0,05}=5,14\right)$. Aplicou-se também o teste de Tukey para determinar as 
diferenças entre os diferentes grupos. Comparando o grupo de 3\% de RFAP com a mistura de referência foi obtido que $\mathrm{p}=0,0032<\mathrm{p}=0,05$. A comparação entre o grupo das misturas de CBUQ com RFPA e com a mistura CBUQ de referência resultou em $p=0,0032<p=0,05$. Destaca-se ainda que as diferenças entre o grupo das misturas com RFPA foram significativas, conforme apontado pela comparação $(p=0,009<p=0,05)$. Estes resultados revelam um incremento significativo na estabilidade quando o fíler é substituído pelo pó de RFPA.

Para o caso de F, existiu uma diminuição na média de $12.9 \%$ para a mistura de $3 \%$ e um aumento de 19,2\% da mistura com $6 \%$ de RFAP com respeito à amostra de CBUQ de referência e, também, com significância estatística de $\mathrm{F}=48,34>\mathrm{F}_{0,05}=5,14$. Estas diferenças dos resultados também demonstraram-se significativas entre todos os grupos. $\mathrm{O}$ resultado $\mathrm{p}=0,02<\mathrm{p}=0,05$ corresponde à comparação entre o grupo da mistura de CBUQ de referência com o grupo da mistura CBUQ-3 RFAP. A comparação da mistura de CBUQ de referência com a de CBUQ- RFAP $6 \%$ resultou em $\mathrm{p}=0,003>\mathrm{p}=0,05$, indicando que não se pode considerar essas diferenças como significativas. Por sua vez, a comparação entre os grupos das misturas CBUQ com RFAP, apontou diferenças ( $\mathrm{p}=0,00016<\mathrm{p}=0,05)$.

Finalmente, os resultados das médias da relação E/F sinalizaram um aumento de 55,3\% para misturas CBUQ-3 RFAP e de apenas 3,6\% para as misturas CBUQ-6 RFAP, ao serem comparadas com a mistura CBUQ de referência. Essas diferenças foram significativas $\left(F=115,68>F_{0,05}=5,14\right)$. A diferença entre as médias da mistura CBUQ de referência e a mistura CBUQ-3 RFAP foi significativa, conforme validado pelo teste de Tukey $(\mathrm{p}=0,000025<\mathrm{p}=0,05)$. No entanto, não existiram diferenças entre as médias das misturas CBUQ-6 RFAP e da mistura de referência $(\mathrm{p}=0,687<\mathrm{p}=0,05)$. Esses resultados apontam uma possível perda de adesão entre o esqueleto mineral e o ligante asfáltico quando o fíler natural é totalmente substituído pelo pó de RCA.

\subsection{Resistência à Tração indireta (RT)}

Os resultados da RT para as misturas são apresentados na Figura 5. Segundo os resultados, pode-se evidenciar que as misturas com RFAP apresentaram maior RT em relação à mistura de referência, destacando-se um melhor comportamento na mistura com 3\% de RFAP. Os resultados apresentados podem apontar que a presença de RFAP contribuiu para aumento da adesividade da mistura, possivelmente, pelas interações físicoquímicas de superfície, conforme descrito nas análises dos resultados do ensaio Marshall. Esse comportamento foi apresentado apesar do aumento do $\mathrm{Vv}$ e da redução do RBV. Vale ressaltar, que as três misturas analisadas apresentaram o mesmo teor de asfalto de 5,3\%. Para analisar se as variações apresentadas eram representativas, foram realizados tratamentos estatísticos conforme apresentado na Tabela 6.

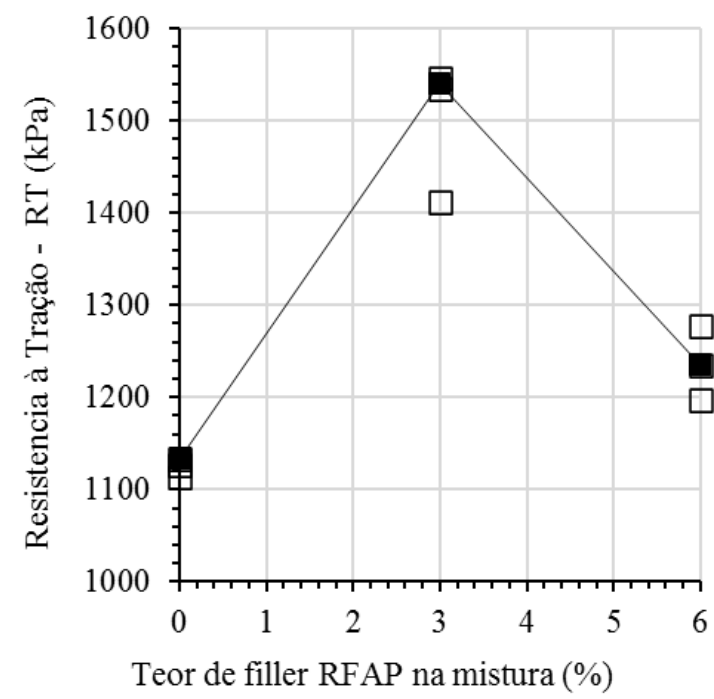

Figura 5: Resultados da resistência à Tração. 
Tabela 6: Estatística do ensaio RT.

\begin{tabular}{l|l|l|l|l|l}
\hline PARÂMETRO & MISTURA & MÉDIA & DESVIO PADRÃO & P & NORMALIDADE SHAPIRO-WILK \\
\hline \multirow{4}{*}{ RT $(\mathrm{kPa})$} & CBUQ Referência & 1124,57 & 10,11 & 0,71 & Sim \\
\cline { 2 - 7 } & CBUQ-3 RFPA & 1495,50 & 71,93 & 0,07 & Sim \\
\cline { 2 - 6 } & CBUQ-6 RFPA & 1236,27 & 40,06 & 0,95 & Sim \\
\hline
\end{tabular}

NaTabela 6, são expostos os parâmetros estatísticos dos grupos de amostras submetidos ao ensaio de RT. Todos os grupos apresentaram distribuição normal nos resultados. A mistura CBUQ-3 RFAP incrementou a média da RT em 33\%, ao ser comparada com a mistura de referência e diante do incremento de $9 \%$ para misturas CBUQ-6 RFAP. O valor da prova ANOVA $\left(\mathrm{F}=47,35>\mathrm{F}_{0,05}=5,14\right)$ mostrou diferenças significativas. Ao aplicar o teste de Tukey essa diferença foi apenas observada entre a mistura de referência e a CBUQ$3 \operatorname{RFAP}(\mathrm{p}=0,002<\mathrm{p}=0,05)$. As diferenças entre a mistura CBUQ-6 RFAP e a mistura de referência não puderam ser consideradas significativas $(\mathrm{p}=0,111>\mathrm{p}=0,05)$.

\subsection{Ensaio Cântabro}

Os resultados da perda de massa das misturas avaliadas e a prova de normalidade são detalhados na Tabela 7 . Embora perceba-se que há uma perda de massa maior nas misturas CBUQ-3 RFAP, esta evidência não teve significância estatística como demostrado pelos valores de ANOVA $\left(\mathrm{F}=1,303<\mathrm{F}_{0,05}=5,14\right)$. A partir desses resultados, pode-se inferir que não existe desagregação significativa entre o esqueleto mineral e o ligante asfáltico com o incremento da percentagem do pó de RFAP.

Tabela 7: Estatística do ensaio Cântabro.

\begin{tabular}{l|l|l|l|l|l}
\hline PARÂMETRO & MISTURA & MÉDIA & DESVIO PADRÃO & P & SHAPIRO-WILK \\
\hline \multirow{4}{*}{ Resistência a abrasão Cântabro } & CBUQ Referência & 5,43 & 0,15 & 0.63 & Sim \\
\cline { 2 - 7 } & CBUQ-3 RFAP & 5,63 & 0,25 & 0.78 & Sim \\
\cline { 2 - 7 } & CBUQ-6 RFAP & 5,31 & 0,31 & 0.061 & Sim \\
\hline
\end{tabular}

\section{CONCLUSÕES.}

Este artigo avaliou a influência da substituição do fíler natural pelo RFAP em misturas asfálticas. Para tal fim, foram produzidas três misturas: uma de referência com materiais convencionais, e as outras com 3\% e 6\% de RFAP em substituição do fíler natural. As misturas foram submetidas a vários testes mecânicos, a partir dos quais, conclui-se que:

- O material RFAP pode ser utilizado em qualquer destas proporções sem que os parâmetros volumétricos (Va, VAM e RVB) ultrapassem as especificações. No entanto, percebe-se um incremento no volume de vazios ao aumentar a percentagem de RFAP, o que pode indicar um aumento na absorção da mistura. Da mesma maneira, não há detrimento dos parâmetros mecânicos estudados (estabilidade e resistência à tração indireta) em função do teor de RFAP utilizado, se comparado com os valores da especificação;

- Os parâmetros mecânicos do ensaio Marshall apontam um aumento significativo na estabilidade e redução da fluência para a mistura elaborada com $3 \%$ de RFAP, o que se traduz em aumento do coeficiente E/F. Embora a mistura CBUQ-6 RFAP apresente comportamento similar nestes parâmetros, demostrou-se com testes estatísticos que a diferença não pode ser considerada significativa; Esses resultados são coerentes com os reportados por Arabani e Azarhoosh [1] quando substitui o total do fíler convencional por RCA.

- Os resultados do ensaio de resistência à tração evidenciam um aumento nas misturas com RFAP se comparado com a mistura de referência. Desde o ponto de vista estadístico, os resultados obtidos com a mistura CBUQ-3 RFAP são significativos, enquanto que a mistura CBUQ- 6RFAP não se consideram significativos. $\mathrm{O}$ anterior evidencia um aumento da aderência entre os agregados e o ligante asfáltico nas misturas com RFAP, o qual pode ser atribuído a presença de oxido de cálcio do RFAP.

- O ensaio Cântabro não trouxe um resultado significativo da perda de massa das misturas o que corrobora com o uso do RFAP como material de enchimento no esqueleto mineral;

- A utilização de RFAP pode ser considerada uma técnica viável desde o ponto de vista técnico e ambiental por permitir uma forma de disposição final aos resíduos RCA, e contribuir à preservação e conservação do meio ambiente em termos do gerenciamento de resíduos sólidos industriais. Porém, estudos adicionais deverão ser realizados com o fim de avaliar o comportamento mecânica mistura ante cargas dinâmicas (modulo resiliente, modulo dinâmico, fatiga e deformação permanente), assim como também o estudo ante a ação de cargas estáticas. Tais avaliações precisarão considerar diversos conte- 
údos de RFAP, fazendo incorporações em massa e volume.

\section{AGRADECIMENTOS}

Os autores agradecem à Universidade Católica de Colômbia pelo apoio financeiro desta pesquisa, mediante o Projeto de titulado "Estudio de mesclas asfálticas com llenantes minerales alternativos. Fase II" CON0000342/2020.

\section{BIBLIOGRAFIA}

[1] ARABANI, M., AZARHOOSH, A.R. "The effect of recycled concrete aggregate and steel slag on the dynamic properties of asphalt mixtures," Constr. Build. Mater., v. 35, pp. 1-7, 2012.

[2] AFSHAR, T., DISFANI, M.M., ARULRAJAH, A., et al. "Impact of particle shape on breakage of recycled construction and demolition aggregates,” Powder Technol., v. 308, pp. 1-12, Feb. 2017.

[3] EU, “EU 2019.” p. https://ec.europa.eu/eurostat/data/database, 2019.

[4] ZHENG, L., et al., "Characterizing the generation and flows of construction and demolition waste in China," Constr. Build. Mater., v. 136, pp. 405-413, 2017.

[5] ROBAYO SALAZAR, R.A., MATTEY CENTENO, P.E., SILVA URREGO, Y.F., et al. "Los residuos de la construcción y demolición en la ciudad de Cali: un análisis hacia su gestión, manejo y aprovechamiento," Revusta Tecnura, v. 19, n. 44, pp. 157-170, 2015.

[6] LEITE, F.D.C., MOTTA, R.D.S., VASCONCELOS, K.L., et al., "Laboratory evaluation of recycled construction and demolition waste for pavements," Construction and Building Materials, v. 25, n. 6. pp. 2972-2979, 2011

[7] FARIAS, M.M., GÓMEZ, A., QUIÑONES, F. "Use of Recycled Aggregates from Construction and Demolition Wastes for the Construction of Flexible Pavements," in Third International Conference on Geotechnique, Construction Materials and Environment., 2013, no. Sep. 2015.

[8] BRASILEIRO, L.L., MATOS, J.M.E. "Revisão bibliográfica: reutilização de resíduos da construção e demolição na indústria da construção civil,” Cerâmica, v. 61, n. 358, pp. 178-189, Jun. 2015.

[9] GÓMEZ, A.M.J. "Comportamento mecânico de um agregado reciclado a partir de resíduos de construção e demolição submetido a carregamentos cíclicos," Universidade de Brasília, 2016.

[10] BARBUDO, A., JIMÉNEZ, J.R., AYUSO, J., et al. "Catalogue of Pavements with Recycled Aggregates from Construction and Demolition Waste," Proceedings, v. 2, n. 20, p. 1282, Oct. 2018

[11] BASTIDAS-MARTÍNEZ, J.G., RONDÓN-QUINTANA, H., ZAFRA-MEJÍA, C. "Study of Hot Mix Asphalt Containing Recycled Concrete Aggregates That Were Mechanically Treated With a Los Angeles Machine," Int. J. Civ. Eng. Technol., v. 10, n. 10, pp. 226-243, 2019.

[12] WONG, Y.D., SUN, D.D., LAI, D. "Value-added utilisation of recycled concrete in hot-mix asphalt," Waste Manag., v. 27, pp. 294-301, 2007.

[13] BHUSAL, S., LI, X., WEN, H. "Evaluation of Effects of Recycled Concrete Aggregate on Volumetrics of Hot-Mix Asphalt,” Transp. Res. Rec. J. Transp. Res. Board, v. 2205, pp. 36-39, 2011.

[14] FARIAS, M.M., SINISTERRA, F.Q., RONDÓN, H.A.Q. "Behavior of a hot mix asphalt made with recycled concrete aggregate and crumb rubber," Can. J. Civ. Eng., v. 46, n. 6, pp. 544-551, Jun. 2019.

[15] GUL, W.A., GULER, M. "Rutting susceptibility of asphalt concrete with recycled concrete aggregate using revised Marshall procedure," Constr. Build. Mater., v. 55, pp. 341-349, 2014.

[16] MOTTER, J.S., MIRANDA, L.F.R., BERNUCCI, L.L.B. "Performance of hot mix asphalt concrete produced with coarse recycled concrete aggregate," J. Mater. Civ. Eng., v. 27, n. 11, pp. 1-7, 2015.

[17] RADEVIĆ, A., ĐUREKOVIĆ, A., ZAKIĆ, D., et al. "Effects of recycled concrete aggregate on stiffness and rutting resistance of asphalt concrete," Constr. Build. Mater., v. 136, pp. 386-393, Apr. 2017.

[18] RAFI, M.M., QADIR, A., SIDDIQUI, H. "Experimental testing of hot mix asphalt mixture made of recycled aggregates," Waste Manag. Res., v. 29, n. 12, pp. 1316-1326, 2011.

[19] ÁlVAREZ, D.A., AENLlE, A.A., TENZA-ABRIL, A.J., et al. "Influence of partial coarse fraction substitution of natural aggregate by recycled concrete aggregate in hot asphalt mixtures," Sustain., v. 12, n. 1, 2020.

[20] EL-TAHAN, D., GABR, A., EL-BADAWY, S., et al., "Evaluation of recycled concrete aggregate in asphalt mixes," Innov. Infrastruct. Solut., v. 3, n. 1, p. 20, Dec. 2018.

[21] GOPALAM, J., PRAKASH GIRI, J., PANDA, M. "Effect of filler on bituminous base layer containing recycled concrete aggregates," Int. J. Transp. Sci. Technol., no. xxxx, 2020.

[22] PASANDÍN, A.R., PÉREZ, I. "Performance of hot-mix asphalt involving recycled concrete aggregates," Int. J. Pavement Eng., v. 0, n. 0, pp. 1-13, 2018.

[23] GIRI, J.P., PANDA, M., SAHOO, U.C. "Use of waste polyethylene for modification of bituminous 
paving mixes containing recycled concrete aggregates," Road Mater. Pavement Des., v. 21, n. 2, pp. 289309, 2018.

[24] MILLS-BEALE, J., YOU, Z. "The mechanical properties of asphalt mixtures with Recycled Concrete Aggregates," Constr. Build. Mater., v. 24, n. 3, pp. 230-235, 2010.

[25] NWAKAIRE, C.M., YAP, S.P., ONN, C.C., et al. "Utilisation of recycled concrete aggregates for sustainable highway pavement applications; a review," Constr. Build. Mater., vol. 235, p. 117444, 2020.

[26] PASANDÍN, A.R., PÉREZ, I. "Adhesion of recycled concrete aggregates, demolition debris, and asphalt," Pet. Sci. Technol., v. 32, n. 21, pp. 2584-2591, 2014.

[27] MIKHAILENKO, P., RAFIQ KAKAR, M., PIAO, Z., et al. "Incorporation of recycled concrete aggregate (RCA) fractions in semi-dense asphalt (SDA) pavements: Volumetrics, durability and mechanical properties," Constr. Build. Mater., v. 264, p. 120166, 2020.

[28] CARDOSO, R., SILVA, R.V., BRITO, J., et al., "Use of recycled aggregates from construction and demolition waste in geotechnical applications: A literature review," Waste Manag., v. 49, pp. 131-145, 2016.

[29] CHEN, M., LIN, J., WU, S. "Potential of recycled fine aggregates powder as filler in asphalt mixture," Constr. Build. Mater., v. 25, n. 10, pp. 3909-3914, 2011.

[30] ASTM C188-09, "Standard test method for density of hydraulic Cement." West Conshohocken, PA, 2009.

[31] INVIAS, "Instituto Nacional de Vías - INVIAS. Especificaciones Generales para Construcción de Carreteras. Bogotá D.C., Colombia.," 2013.

[32] AASHTO T 96-02, "Standard Method of Test for Resistance to Degradation of Small-Size Coarse Aggregate by Abrasion and Impact in the Los Angeles Machine." American Association of State and Highway Transportation Officials, p. 12, 2019.

[33] AASHTO T 327, "Standard Method of Test for Resistance of Coarse Aggregate to Degradation by Abrasion in the Micro-Deval Apparatus." American Association of State and Highway Transportation Officials, p. 9, 2012.

[34] NLT-354/91, “Indices de lajas y agujas de los áridos para carreteras.” CEDEX, p. 4, 1991.

[35] ASTM D5821, "Standard Test Method for Determining the Percentage of Fractured Particles in Coarse Aggregate.” ASTM International, West Conshohocken, p. 5, 2018.

[36] AASHTO T 104-99, "Standard Method of Test for Soundess of Aggregate by Use of Sodium Sulfate or Magnesium Sulfate.” American Association of State and Highway Transportation Officials, p. 11, 2016.

[37] DNER-ME 078-94, "DNER-ME 078: Agregado graúdo - adesividade a ligante betuminoso." Departamento Nacional de Estradas de Rodagem, Rio de Janeiro, p. 3, 1994.

[38] AASHTO T 84-00, "Standard Method of Test for Specific Gravity and Absorption of Fine Aggregate." American Association of State and Highway Transportation Officials, p. 9, 2004.

[39] AASHTO T 85-91, "Standard Method of Test for Specific Gravity and Absorption of Coarse Aggregate." American Association of State and Highway Transportation Officials, p. 10, 2004.

[40] ASTM D5 / D5M, "Standard Test Method for Penetration of Bituminous Materials." ASTM International, West Conshohocken, PA, p. 4, 2019.

[41] ASTM D36 - 95, "Standard Test Method for Softening Point of Bitumen (Ring-and-Ball Apparatus)." ASTM International, West Conshohocken, p. 4, 2000.

[42] NLT 181/88, "Indice de penetracion de los betunes asfálticos.” CEDEX, p. 2, 1988.

[43] AASHTO T 228, "Standard Method of Test for Specific Gravity of Semi-Solid Asphalt Materials." American Association of State and Highway Transportation Officials, p. 3, 2009.

[44] AASHTO T-316, "Standard Method of Test for Viscosity Determination of Asphalt Binder Using Rotational Viscometer." American Association of State Highway and Transportation Officials, p. 10, 2019.

[45] ASTM D3143, "Standard Test Method for Flash Point of Cutback Asphalt with Tag Open-Cup Apparatus.” ASTM International, West Conshohocken, p. 6, 2008.

[46] ASTM D113, "Standard Test Method for Ductility of Asphalt Materials." ASTM International, West Conshohocken, PA, p. 5, 2017.

[47] ASTM D2872, "Standard Test Method for Effect of Heat and Air on a Moving Film of Asphalt (Rolling Thin-Film Oven Test).” ASTM International, West Conshohocken, PA, p. 12, 2019.

[48] IDU 620-18, "Mezcla asfáltica en caliente densa, semidensa y gruesa." Instituto de Desenvolvimento Urbano, Bogota D.C., 2019.

[49] SENA NETO, P.G., AMORIM, E.F., INGUNZA, M.P.D. "Análises de dosagens de concreto asfáltico do tipo Pré Misturado a Frio (PMF) utilizando Resíduos da Construção e Demolição de obras (RCD)," Matéria (Rio Janeiro), v. 24, n. 4, 2019.

[50] CUNHA, C.B., BUDNY, J., KLAMT, R.A., et al., "Avaliação de concreto asfáltico usinado a quente com incorporação de material fresado," Matéria (Rio Janeiro), v. 23, n. 3, Oct. 2018. 
[51] ASTM D7064, "Standard Practice for Open-Graded Friction Course (OGFC) Mix Design." ASTM International, West Conshohocken, PA, p. 6, 2004.

[52] BASTIDAS-MARTÍNEZ, J.G., CARVALHO, J.C., LUCENA, L.C., et al., "Preliminary study of hot mix asphalt containing water treatment and sewage sludge," Arch. Civ. Eng., v. 66, n. 1, pp. 225-240, 2020.

[53] CALA, A., CARO, S., LLERAS, M., et al., "Impact of the chemical composition of aggregates on the adhesion quality and durability of asphalt-aggregate systems," Constr. Build. Mater., v. 216, pp. 661-672, 2019.

[54] MUNIANDY, R., ABURKABA, E., TAHA, R. "Effect of mineral filler type and particle size on the engineering properties of stone mastic asphalt pavements," J. Eng. Res., v. 10, n. 2, pp. 13-32, 2013.

\section{ORCID}

Juan Gabriel Bastidas-Martínez

Juan Carlos Ruge Cárdenas

Liosber Medina García

Robinson Andrés Giraldo Zuluaga https://orcid.org/0000-0002-6818-0322

https://orcid.org/0000-0002-9100-6058

https://orcid.org/0000-0002-4091-3491

https://orcid.org/0000-0001-6674-1977 